\title{
Rechtsgeschichte
}

\section{Hubert Rottleuthner}

»Lüritz gibt es nicht« 
baren Kompromiss zu unterbreiten. Dann wird auch die gefügige Mitarbeit der Bürgerlichen an einer Verfassung verständlicher, die durch das Fehlen zuverlässiger Kontrollen sehr bald die Konzentration aller Macht in den Händen der Partei erlaubte. Auch die Juristen unter den Autoren der Verfassung scheinen ihre Fallstricke gar nicht richtig zur Kenntnis genommen zu haben. Vor allem die Ablehnung der Gewaltenteilung durch die SED, der alte Slogan "alle Macht den Sowjets", hätte nach den Erfahrungen mit dem Stalinismus in der UdSSR doch eigentlich nicht nur bei den bürgerlichen Parteien, sondern auch bei ihren Kollegen von der ehemaligen SPD Argwohn erregen müssen. Die Einmütigkeit bei den Abstimmungen im Verfassungsausschuss kann ich mir nur damit erklären, dass auch seine bürgerlichen Mitglieder es in diesen frühen Jahren zum Mindesten für möglich hielten, dass ein sozialistisches Verfassungsmodell vielleicht doch Gewähr für eine bessere Zukunft bieten könne. Im Spiegelbild zu den Reaktionen in Westdeutschland mögen sie ihrerseits nicht verstanden haben, warum man im Westen nicht ein offeneres Ohr für ihre Vorschläge zur Erneuerung Deutschlands hatte. Hatte die deutsche Berufsbeamtenschaft der Hitlerdiktatur nicht gerade so willfährig gedient wie zuvor dem Kaiser? Abschaffen! Waren die von

\section{»Lüritz gibt es nicht «*}

Was kann einem die Sicherheit verschaffen, vom Besonderen und nicht vom Sonderlichen auf das Allgemeine zu schließen? Da tat sich nach I990 ein Aktenfund im Holzkeller eines vormaligen DDR-Gerichts auf; aber kann man mit einer Ministerialbürokratie ernannten Richter nicht willfährige Erfüllungsgehilfen des Dritten Reiches gewesen? Richter in Zukunft wählen lassen! Sollten nicht auch in Hessen und Bremen die wichtigsten Produktionsmittel verstaatlicht werden? Der Sozialismus muss in diesen Jahren in der Luft gelegen haben. So konnte zum Beispiel Karl Schultes, Jurist und Staatsrechtslehrer, der schon 1950 nach West-Berlin fliehen sollte und der von I 964 bis I 976 Verfassungsrichter in Nordrhein-Westfalen war (236), noch im September 1948 den Vorschlag machen, Artikel 6 der Verfassung als unmittelbar anwendbare Strafbestimmung zu konzipieren (203) das heißt: Grundrechte nicht als Schutzschild der Bürger gegen den Staat, sondern als Waffe des Staates zur Disziplinierung seiner Bürger zu verstehen. Was ging in seinem Kopf vor? Nach dem Untergang des Hitler-Reichs muss der Glaube an die Machbarkeit einer besseren und gerechteren Welt verführerisch genug gewesen sein, um die juristischen Instinkte auch bürgerlicher Verfassungsschöpfer für eine kurze Zeit lang einzulullen.

Aber das sind die Spekulationen einer Rezensentin. Heike Amos' beachtliche Arbeit hat uns mit den Fakten versehen, die sie möglich machen.

Inga Markovits

Verfahrensakten eines Kreisgerichts (KG) eine Justizgeschichte für das ganze Land schreiben? Es blieb allerdings nicht bei den Verfahrensakten und auch Generalakten des KG »Lüritz«. Es tauchten Haftbefehle vor Ort auf; Akten des be-

\footnotetext{
* Inga Markovits, Gerechtigkeit

in Lüritz. Eine ostdeutsche

Rechtsgeschichte, München:

C. H. Beck 2006, 303 S.,

ISBN 978-3-406-55054-6
} 
nachbarten KG und Bezirksgerichts (BG) »Neuburg « und Bestände anderer Archive konnten genutzt werden. Hinzu kam die Lektüre von Jahrzehnte alten Zeitungsmeldungen. Diese Papierflut wurde belebt durch zahlreiche Gespräche mit noch lebenden (und gesprächsbereiten) Justizjuristen, darunter ehemalige Volksrichter und Richter(innen) der letzten DDR-Jahre, auch mit Richtern des Bezirksgerichts, Rechtsanwälten, Mitarbeitern der Kreis- und Bezirksleitung der SED, Stadtbeamten, Mitarbeitern der Staatssicherheit, auch Klägern, Beklagten, Angeklagten.

Dieses lokal begrenzte Material wurde ergänzt durch Rückgriff auf vorhandene Untersuchungen (z. B. zur »Aktion Rose« [49 f.]), auf Dokumente zentraler Justizorgane (vor allem des Obersten Gerichts [OG] und des Ministeriums der Justiz [MdJ]). Ohne das reiche KontextWissen zum DDR-Recht, über das Markovits seit Jahrzehnten verfügt, wäre das lokale Material nur schwer zu interpretieren gewesen.

Den Schluss über »Lüritz « hinaus auf »Ostdeutschland « rechtfertigt Markovits vor allem mit dem Bemühen der zentralen Justizorgane um eine »Einheitlichkeit der Rechtsprechung « (I 2 f.). Die Homogenität der DDR-Justiz, insbesondere was die Strafzumessung angeht, war gewiss erheblich größer als in der BRD, in der die Unabhängigkeit der Richter homogenisierende Maßnahmen der Justizverwaltung, aber auch z. T. der oberen Instanzen nahezu unmöglich macht. Man stelle sich für die BRD eine Untersuchung des AG Lübeck für die letzten 50 Jahre vor - vorausgesetzt, die Aktenordnung wäre nicht eingehalten worden und es gäbe noch solche Papierberge! Das Vorgehen ähnelt dem des soziologischen Klassikers aus den 5oer Jahren (lange vor allen möglichen Regional- oder Lokalstudien) »Ein Dorf in der Vaucluse« von
Laurence Wylie, der damit Aufschluss für die ganze französische Gesellschaft gewinnen $\mathrm{zu}$ können glaubte.

Sicherlich, für versuchte Republikflucht gab es ziemlich einheitliche Tarife und wie mit Ausreisern umzugehen war, wurde auch bald von oben angeleitet (88) - alle Kreisgerichte, behauptet Markovits, hätten diese Politik ohne Zögern mitgemacht (was plausibel klingt, aber im Rahmen ihrer Untersuchung gar nicht belegt werden kann). Aber es gibt auch andere Bereiche, in denen das nicht so geschah, trotz Leitungsdokumenten (z. B. zum Entzug des Erziehungsrechts). Von einigen Fällen kann man fast schon a priori sagen, dass sie vor einem KG gar nicht auftauchen konnten. So stellt Markovits fest, dass politisch brisante Sachen gleich an das BG »Neuburg « oder das dortige KG gingen (I23) (das seien aber seit I 968 nur I-2\% der "politischen « Fälle gewesen); Verfahren wegen »Boykotthetze« (Art. 6 DDR-Verf. I949) wurden nicht in »Lüritz «, sondern in »Neuburg « durchgeführt. In frühen Jahren beschäftigte sich auch gleich das OG damit. Zur Tätigkeit der gesellschaftlichen Gerichte muss auf andere Studien zurückgegriffen werden (64). Es bleiben nur die wenigen Berufungen gegen Entscheidungen der Konfliktkommissionen, die vor dem KG landeten.

In »Lüritz « habe sie keinen Fall von "Zwangsadoption « in der Zeit zwischen I965 und 1989 gefunden (I07). In »Neuburg «, geschweige denn in »Lüritz«, sei kein Todesurteil $\mathrm{zu}$ finden gewesen (I44): quod non erat in Lüritz, non erat in DDR? Was schließlich das Ende des KG »Lüritz « angeht, so ist der Schluss vom Allgemeinen auf das Einzelne angebracht: Mit der DDR ging auch dieses Gericht unter.

Markovits schreibt eine »Rechtsgeschichte von unten « - aber eher eine von der unteren Instanz her, von der sie auf die gesamte DDR- 
Justizlandschaft schließen möchte. Wenn es schon problematisch ist, von "Lüritz « auf das Recht der DDR zu schließen, dürfte ein Schluss von Gerichtsakten - auch wenn DDR-Akten viel informativer sind als die aus der BRD, was den Verfahrensablauf angeht - auf das Alltagsleben und das in der Gesellschaft »lebende Recht « ausgeschlossen sein: auf die Rechtspraxis in Betrieben, Familien, aber auch hinsichtlich des kriminellen Dunkelfeldes (95 f.).

In der gesamten Arbeit bleibt unklar, welche Auswahl aus dem Material, insbesondere den Verfahrensakten, getroffen wurde. Nicht der deutsche, aber der amerikanische Leser konnte erfahren, dass nur jeder dritte bis fünfte Jahrgang vollständig durchgesehen wurde (in ereignisreichen Zeiten war es wohl jeder dritte). ${ }^{\mathrm{I}}$ Hinsichtlich der Grundgesamtheit erfahren wir, dass zivilrechtliche Akten ab 1945/46 existieren - es fehlen allerdings Akten um das Jahr 1948 herum (47); Strafrechtsakten seien ab I 952 vorhanden (2I). Die Akten des BG "Neuburg " standen ab I968 zur Verfügung (I44). Wir erfahren aber nichts über den quantitativen Umfang der Grundgesamtheit oder der Stichprobe. Es heißt einmal, dass in den letzten Jahren am KG »Lüritz « fünf Richter pro Jahr Iooo Sachen entschieden hätten (9); das Strafrecht habe mit 400-500 Angeklagten etwa ein Drittel der Geschäftsbelastung ausgemacht (I 23).

Wer das Narrative in historischen Arbeiten schätzt, wird mit dem vorliegenden Buch auf seine Kosten (von I9,90 Euro) kommen. Und die Autorin erzählt empathisch, scheut sich aber auch nicht vor kräftigen Wertungen (»Ich mag ihn nicht. « [3 I]). Wer allerdings Justizforschung nicht in Erzählungen auflöst, sondern die Rechtsprechung auch an kleineren Gerichten als ein Massengeschäft versteht, das ohne Routinen und Trivialisierungen nicht auskommt, wird weitere
Anforderungen stellen. Allein schon zur Fundierung des Schlusses von "Lüritz « auf die DDR hätte es nahe gelegen, einen justizstatistischen Abgleich vorzunehmen, d. h. eine Gegenüberstellung der Geschäftsstatistiken von »Lüritz« mit denen der DDR-Justiz.

In den Kapiteln des Buches, in denen einzelne Rechtsgebiete behandelt werden - Eigentum, Familie, Strafen -, wimmelt es von eingestreuten justizstatistischen Informationen, mal für das KG »Lüritz «, mal für die DDR insgesamt, und dies für nach unerfindlichen Kriterien ausgewählte einzelne Jahrgänge. Es werden Behauptungen über langfristige Entwicklungen aufgestellt, deren Daten-Basis man doch gerne etwas aufgehellt hätte. Ist das Wissen aus internen Gerichtsstatistiken gewonnen oder wurde wirklich z.B. jede Strafverfahrensakte durchgegangen, um die These (5I) über die Jahrzehnte umspannende Änderung in der Relation von Delikten gegen das gesellschaftliche und das private Eigentum zu stützen? (Und das ohne EDV-Einsatz [I63].) Wurden alle Kündigungsschutzklagen erfasst als Voraussetzung für die Behauptung (66), dass fast alle diese Klagen von I 949 bis I 989 erfolgreich waren?

In dem weiteren Kapitel über die Rolle der Partei wird das Problem eines lokalen und "niedrigen " Ansatzes noch einmal deutlich. Die Verfahrensakten geben (verständlicherweise) kaum etwas her über Steuerungsaktivitäten der Parteistellen, allenfalls in den frühen Jahren. Die »operative " Leitungstätigkeit ist nur unzureichend in Akten dokumentiert. Die Mechanismen, über die der Einfluss der Partei auf die Rechtsprechung gesichert wurde, sind mittlerweile recht gut erforscht (knapp zusammengestellt auf S. I 89 ff., I99f.) - freilich geschah das in den entsprechenden Untersuchungen mit Blick "von oben ", wie es einer Steuerungsperspektive zunächst

I Vgl. Inga Markovits, Justice in

Lüritz, in: The American Journal

of Comparative Law 50 (2002)

819-874, 823 . 
auch angemessen ist. Markovits kann nun eine Reihe von Fällen auf der unteren Ebene beisteuern - weniger aus Prozessakten als aus Gesprächen mit Parteifunktionären (z. B. I95 f.); und sie konnte sich auch Materialien der Kreisund Bezirksleitung bedienen (I 82). Allerdings werden hier auch Leitungsdokumente und operative Tätigkeiten des MdJ oder des BG hineingemischt: Die Abhängigkeit der Justiz von der Partei sei zunehmend durch ihre Abhängigkeit von der Justizbürokratie ersetzt worden. Aber die gab es schon sehr früh in Gestalt der Justizverwaltungsstellen (2II). Die Partei sei später nur noch "Spinne " gewesen - kontrollierend auf der Lauer liegend (225).

Mit Recht legt Markovits großes Gewicht auf das Phänomen der Asozialen (I 55 ff., $262 \mathrm{ff}$.). Zum einen sind sie quantitativ von großem Gewicht. Nachdem der alte $\mathbb{S} 36 \mathrm{I}$ des RStGB I 968 abgelöst wurde durch $\$ 249$ StGBDDR, ergingen in den 7 oer Jahren die meisten Haftbefehle wegen Asozialität (I30); I988 machten die »Asozialen « fast ein Viertel der Strafgefangenen in der DDR aus (162). - Im Schnittbereich von Asozialität und Entzug des Erziehungsrechts macht Markovits die meisten Unrechtsurteile aus (III); hier findet sie auch das ihrer Ansicht nach unmenschlichste Urteil ( I 2 ff.). - Schließlich kann man an der Gruppe der als »asozial « Stigmatisierten Grundcharakteristika der DDR und ihrer Justiz deutlich machen. Neben den "Ausreisern « stellten sie die zentrale Bedrohung der System-Loyalität dar. Sie waren keine unartigen Kinder, sondern sie hatten den Familienzusammenhalt aufgekündigt. Im Umgang mit ihnen wird das ungeheure Kontrollbedürfnis, die »Erziehungsmanie « des Rechtssystems (I4) deutlich. Es ging um die "Umerziehung des Menschen durch das Recht « (68). Eine »arbeitsbesessene Erziehungsdikta- tur « (I64) operierte mit einem »Rechtssystem, das seine Kinder weder ausscheren ließ noch fallenlassen wollte « (I6I). Mit Hilfe des Rechts sollte gesellschaftliche Solidarität geschaffen werden (250) - aber wohl nicht im Sinne Durkheims.

Hier wäre ein Vergleich mit der frühen BRD einmal sinnvoll. Dort galt bis I969 (nicht bis I959, wie Markovits meint [I 56]) \$36I Ziff. 7 StGB, nach dem Bezieher von öffentlichen Armenmitteln zu Haft (maximal 6 Wochen) verurteilt werden konnten, wenn sie aus »Arbeitsscheu « eine zugewiesene Arbeit verweigerten. $\mathbb{} \$ 2$ d StGB sah nach Verbüßung der Haft die Möglichkeit einer Einweisung in ein Arbeitshaus für 2 Jahre vor. Auch unterhaltspflichtige Sozialhilfeempfänger konnten, wenn sie keine zumutbare Arbeit annahmen, nach $\mathbb{2} 6$ Bundessozialhilfegesetz von I96I bis 1974 in einer "Arbeitseinrichtung « untergebracht werden. Diese Regelung erkannte das Bundesverfassungsgericht als verfassungsgemäß an (Beschluss vom I 5.I2. I970, NJW I97I, 4I9). Hingegen hatte dieses Gericht mit einem Urteil vom I 8.7.1967 (NJW I967, I795 ff.) die Regelungen zur »Hilfe für Gefährdete « in $\mathbb{S}$ 72-74 BSHG für verfassungswidrig erklärt (Willensschwache, Hemmungslose oder Verwahrloste konnten in eine Anstalt eingewiesen werden, wo sie sich an regelmäßige Arbeit und Sesshaftigkeit gewöhnen können sollten), und zwar mit dem Argument, dass es nicht Aufgabe des Staates sei, aus seinen Bürgern bessere Menschen zu machen. Das wäre in nuce der Unterschied des Rechtsverständnisses in Ost und West.

Markovits macht am Ende zwei Entwicklungstendenzen erkennbar (293 f.):

- das Sinken des politischen Glaubens an den Sozialismus, der auf hohem Niveau ansetzte mit der Überzeugung der Volksrichter, einem gerech- 
teren Staat zu dienen (»Volksrichter sind Missionare der sozialistischen Gesetzlichkeit « [33]), aber dann in ideologischen Notlügen und Zynismus endete;

- ein wachsender Glaube an das Recht, ausgehend von einem Zustand der relativen juristischen Unwissenheit bei den Volksrichtern bis zur Heilssuche in Formalität und juristischer Routine im letzten Amtsjahr.

Wie viele andere vor ihr kritisiert auch Markovits die "Geheimhaltungs-Manie « (233) der DDR-Justiz; das Rechtssystem habe keine Öffentlichkeit vertragen können (232). Das trifft freilich nicht nur auf die Justiz zu. Allerdings empfiehlt sich eine soziologisch aufgehellte, vergleichende Betrachtung der jeweils bestehenden Schranken zwischen öffentlich und geheim (nicht öffentlich/privat). In der Definition einer solchen Schranke unterscheiden sich Gesellschaften und politische Systeme; was ist öffentlich, was ist vertraulich, geheim, was ist Verschlusssache etc. Nur selten wird etwas nicht öffentlich gemacht wegen eines schlechten Gewissens oder

\section{Kein Ort. Nirgends*}

Ein »Lüritz « gab es in Ostdeutschland nicht; Gerechtigkeit auch nicht, denn warum sollte es in der DDR anders gewesen sein als im (seit Jahrzehnten) Heimatland von Markovits, in dem seinerzeit ein bis heute mit Recht berühmter Richter auf die gutgemeinte Aufforderung »do justice, Justice! « antwortete » that's not my job « und sich auf den Weg zu seinem Job machte, » of allegedly not doing justice «.

Im vorliegenden Fall handelt es sich um eine Rechtsgeschichte von unten. Markovits bietet aus Scham; es sind eher Kontrollbedürfnis oder paternalistische Fürsorge, um die lieben (Landes-)Kinder nicht zu beunruhigen. - Markovits betreibt selbst Geheimniskrämerei mit dem Pseudonym ihres Gerichtsortes - diesmal aus Rücksichtnahme für ihre auch pseudonymisierten Informanten. (Die Lösung für den "wahren« Gerichtsort kann man als Kreuzworträtsel aufgeben: Kreis- und Hafenstadt in der DDR mit 6 Buchstaben - Merke: »Lüritz gibt es nicht. « (I) Dann lässt sich auch leicht sagen, wo sich das Bezirksgericht "Neuburg " - Hafenstadt mit 7 Buchstaben - befand.)

Auch in dem soziologischen Klassiker über ein Dorf in der Vaucluse bediente sich der Verfasser eines verschlüsselten Namens. Aber sehr bald kam heraus, dass es sich um Roussillon handelte - was zu einem ungeheuren Touristenstrom, nicht nur von amerikanischen Soziologen, führte. Ein ähnlicher Gerichts-Tourismus steht der Hafenstadt wohl nicht bevor.

\section{Hubert Rottleuthner}

eine in dieser Weise erstmalig versuchte Verknüpfung von Vergangenheits- und Gegenwartsgeschichte, von oral und von file history. Die Aufstiegs- und Verfallsgeschichte eines Rechtssystems, das mit den Hoffnungen von wenigen begann und mit den Enttäuschungen von vielen endete, vollzog sich für sie - und vollzieht sich nun für den Leser - in den jährlich etwa eintausend straf-, zivil-, arbeits- und familienrechtlichen Fällen im Alltag von, wie gesagt, »Lüritz «, einer im Nordwesten der DDR gelegenen Stadt

\footnotetext{
* Inga Markovits, Gerechtigkeit

in Lüritz. Eine ostdeutsche

Rechtsgeschichte, München:

C. H. Beck 2006, 303 S.,

ISBN 978-3-406-55054-6
} 